\title{
ESTUDIO DE LA GERMINACIÓN DE ONONIS TRIDENTATA L.
}

\author{
Mari Carmen ESCRIBÁ BAEZA y Emilio LAGUNA LUMBRERAS
}

\begin{abstract}
RESUMEN. Estudio de la germinación de Ononis tridentata L. Se han realizado ensayos de germinación con semillas de Ononis tridentata L. especie endémica en la Comunidad Valenciana, sometiéndolas a diferentes tratamientos de escarificación (H2SO4, lejía). El comportamiento más adecuado ha sido el del testigo.
\end{abstract}

Palabras clave. Embrión, escarificación, germinación, plántula, radícula, sésil.

ABSTRACT. Germination study of Ononis tridentata L. Germination behavieur of seeds of Ononis tridentata L., an endemic taxon in the Community Valenciana, was evaluated follouing scarification treatments (H2SO4 and clorox) at constant temperatures. Best results were obtained with the control treatment.

Key words. Embryo, scarification, germination, seedling, radicle, sessile.

\section{INTRODUCCIÓN}

Perteneciente a la familia de las Leguminosae, se localizan en la Comunidad Valenciana contadas poblaciones del táxon vegetal Ononis tridentata, propio de matojares con suelos formados a partir de materiales yesíferos en Valencia y Alicante (GómezCampo, 1987). Ha sido calificada por la UICN como LRlc (menor preocupación), y son numerosas las medidas conservacionistas que se están llevando a cabo actualmente. Entre tales medidas hay que mencionar que su presencia ha potenciado la creación de Microrreservas de flora.

Se trata de un nanofanerófito muy ramificado con hojas cortamente pecioladas, en general trifoliadas y suculentas, foliolos subsésiles cuneados o sublineares, ordinariamente dentados en el ápice (var: tridentata). Flores agrupadas en racimos, sobre pedúnculos glandulosos, cáliz de 6-8 $\mathrm{mm}$., corola papilionada de color rosa. Legumbre pilosa-glandulosa, péndula, con una media de 2 a 3 semillas.

\section{OBJETIVOS}

El objetivo de este estudio es conocer el comportamiento de las semillas de Ononis tridentata frente a diferentes tratamientos. Interesa igualmente estudiar las necesidades de cultivo de la planta y todo cuanto sea posible sobre su desarrollo para poder proporcionarle una protección adecuada. El 
estudio inicial es el de germinación, que termina cuando se inicia la actividad fotosintética (Besnier, 1989).

Las plántulas obtenidas en los ensayos que se describen en el apartado correspondiente, se evaluaron para observar su desarrollo y comportamiento en cultivo. En vivero se procede al endurecimiento progresivo de los ejemplares para su reintroducción en el medio natural; se trata de reforzar las poblaciones de donde se recolectaron las semillas o crear nuevas poblaciones en lugares con características climatológicas y orográficas adecuadas para la supervivencia de la especie. También se destinan a creación de rocallas educativas de flora endémica.

\section{MATERIAL Y MÉTODO}

Se trata de un taxón cuyas semillas son difíciles de recolectar, debido a que aún en estado inmaduro, la semilla es parasitada por larvas de insectos, originando una merma considerable en la formación de semillas; aquellas que consiguen continuar con su formación y desarrollo, caen al suelo al madurar, pues la legumbre va abriendo con la maduración. Así pues para obtener una muestra considerable de semillas maduras, se debe visitar en repetidas ocasiones la población a estudiar.

Una práctica de interés es la de embolsar las legumbres con tela transpirable, de manera que al madurar caen en ella en vez de al suelo y se pueden recolectar. En este caso se empleó una tela llamada tul, bastante flexible y con pequeños agujeros que permiten transpirar a la semilla. Aun efectuando el embolsado, se visitó en repetidas ocasiones la población para recolectar semilla.

Se ha recolectado una muestra considerable de material vegetal de Castillo de Jalance, término municipal de Jalance; fecha recolección 5, 9 y 12 de diciembre 2002. Peso de 100 semillas: 0.0585 gr.

Las semillas recolectadas se introdujeron en bolsas de papel permeable, que se mantuvieron en lugar fresco y seco. Para la limpieza de las semillas se emplearon tamices de diferente luz de malla. Una vez retiradas las impurezas se colocaron en una cámara estanca para su desecación progresiva entre 30 y 45 días, y pasado este tiempo se conservaron hasta el momento del ensayo en cámaras frigoríficas convencionales, cuya temperatura está alrededor de $4{ }^{\circ} \mathrm{C}$.

Llegado el momento de la siembra, se seleccionó el lote de cada partida de semillas a estudiar, se desinfectaron con hipoclorito sódico al $10 \%$ durante 10 minutos y se lavaron con agua abundante. Se sembraron en placa Petri de $9 \mathrm{~cm}$ de diámetro, entre papel de filtro Albet, en lotes de 25 semillas por placa, tal como se indica en la normativa ISTA. Dichas placas se etiquetaron perfectamente y se colocaron en una cámara germinadora modelo MLR-350 de la marca SANYO, en la que se habían preestablecido unas condiciones de temperatura de 20-18 y $10{ }^{\circ} \mathrm{C}$ durante periodos de 8 horas, y un total de 1500 lux durante 10 horas al día. La lectura de la germinación en las placas fue diaria, y el riego con agua destilada, hasta el punto de saturación.

El total de la muestra recolectada se dividió en lotes de los cuales dos de ellos se conservaron a corto y largo plazo, y con los restantes se estableció una serie de ensayos de germinación.

La conservación de lotes de semillas resulta de gran interés, pues además que formar parte del banco de germoplasma de flora endémica, interesa pasado un tiempo realizar ensayos de germinación (tanto con las conservadas a corto como a largo plazo), con objeto de comprobar si hay pérdida de 
poder germinativo con el paso del tiempo, y en caso de haberlo de qué porcentaje se trata; si la conservación es adecuada y si necesitan algún tipo de tratamiento específico.

Para poder establecer una comparación entre los diferentes ensayos se ha establecido un tiempo de duración del ensayo de 31 días.

Con los lotes de semilla se efectuaron los siguientes tratamientos: testigo, escarificación con lejía y escarificación con H2SO4 a concentraciones de 10,15 y $20 \%$.

Los parámetros de interés en ensayos de germinación son los siguientes:

T. in.: tiempo necesario para que comience la germinación; T. 50\%: tiempo (expresado en días) necesario para alcanzar el valor medio del porcentaje de germinación final. Punto T.: momento en que la velocidad de germinación empieza a descender; \% T.: germinación en dicho momento; V.P.: valor pico; \% Gr. Mx.: punto en que la germinación del ensayo es máxima; P.\% Mx.: días necesarios para que se haya alcanzado este punto de germinación máxima; G.M.D.: germinación media diaria; V.G.: valor de germinación;'Nsm sb: número de semillas sembradas de este lote; \% G. Tt: germinación del total de la muestra.

\section{RESULTADOS Y DISCUSIÓN}

Se muestra a continuación la gráfica de germinación parcial diaria (fig. 1) del resultado del ensayo testigo, así como una gráfica comparativa entre vigor de germinación y valor pico (fig. 2). El Valor Pico es el momento en que la velocidad de germinación empieza a descender y se obtiene del cociente entre el porcentaje de germinación en el punto $\mathrm{T}$ y los días necesarios para llegar a este momento $(\mathrm{VP}=$ $\% \mathrm{~T} / \mathrm{T})$. La capacidad germinativa de un lote de semillas indica su poder para formar plántulas con buenas condiciones en campo, y el vigor de germinación es dicha capacidad en condiciones desfavorables para la semilla (Thomson 1979); se obtiene del producto del resultado de GMD por el VP $\mathrm{VG}=\mathrm{GMDxVP}$ ).

También se ofrece una gráfica con los valores de porcentajes de germinación total de los diferentes tratamientos (fig. 3).

Los parámetros obtenidos en este

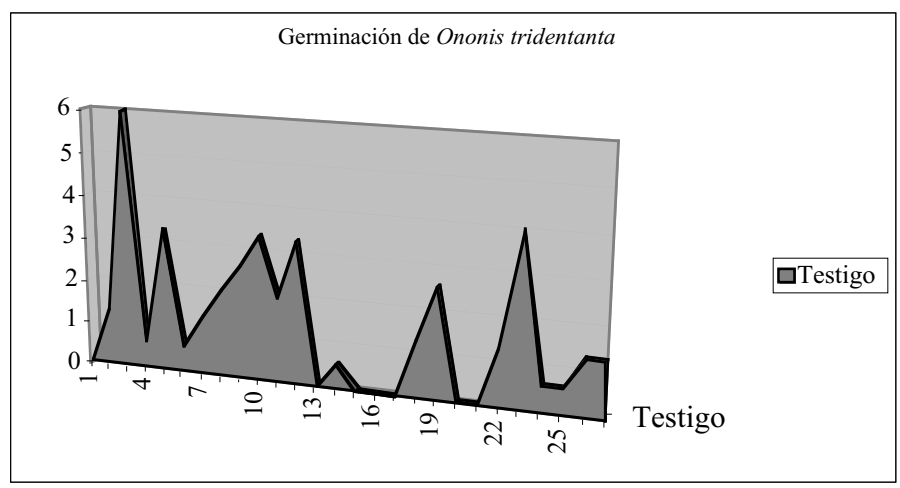

Figura 1. Germinación de Ononis tridentata. Germination Ononis tridentata. 
estudio según los diversos tratamientos efectuados a las semillas quedan reflejados en la tabla.

A la hora de analizar los resultados obtenidos, para interpretar el comportamiento de las semillas, resulta de especial interés observar en conjunto tanto los datos numéricos de los parámetros de germinación como la gráfica resultado del ensayo.

El lote de semillas del tratamiento testigo inicia la geminación rapidamente (en tan solo dos días), siendo el porcentaje total de germinación de 40.6 \%. En ningún otro ensayo se obtuvo este parámetro. También en breve (3 días) se obtiene el pico de germinación Máxima con un $6 \%$, punto algo bajo comparándolo con otras especies estudiadas anteriormente como es el caso de Ononis fruticosa.

El embrión de la semilla de este lote testigo empieza a movilizar demasiado rápido en cuanto tiene condiciones idóneas de temperatura y humedad, de manera que se obtienen picos considerables de germinación al principio del ensayo y hasta el día 13, a partir de entonces las semillas germinan de manera desordenada e irregular con el paso del tiempo. Acabado el ensayo

\begin{tabular}{ll}
\hline Parámetro & Testigo \\
\hline T. in. & 2 \\
T 50\% & \\
Punto T & 3 \\
\% T. & 6 \\
V P & 2 \\
\% G Mx & 3 \\
P \% Mx & 6 \\
GMD & 1.309 \\
VG & 2.618 \\
Nsm sb & 150 \\
\% G Tt & 40.6 \\
\hline
\end{tabular}

Tabla 1. Parámetros de germinación. Germination parameters. el ritmo de germinación media es bastante bueno, alcanza un valor de 1.3. También el valor de V.G. es óptimo, obteniendo un valor numérico de 2.618 que es mucho más elevado en el ensayo testigo que en el resto de ensayos realizados entre los cuales es el de escarificación sulfúrica al 20\% el mejor, que da lugar a un resultado de V.G. de 1.27.

Los tratamientos de escarificación a la semilla con objeto de romper la dureza de la testa que impide el flujo de agua y oxígeno, han sido con lejía y ácido sulfúrico. El uso de ácido sulfúrico como escarificador químico trata de simular los procesos degradativos de la testa que suceden en condiciones naturales (Tran \& Cavanah,1984), y constituye una práctica tan eficaz como conocida (Hopkins, 1923); (Devesa, Ruiz \& Buzo, 2000). Debe tenerse en cuenta que la acción prolongada de este ácido puede ser nociva para las semillas (Madia de Chaluat, 1985).

Se realizó escarificación sulfúrica a diferentes concentraciones, obteniendo un comportamiento en las semillas bastante similar. En todos ellos, la geminación comienza en breve ( 2-3 días). De entre los tres tratamientos hay que destacar el de semillas sometidas al $20 \%$ de $\mathrm{H} 2 \mathrm{SO} 4$, cuyo porcentaje de germinación es más elevado (35\%), también son mucho mejores los parámetros VP y VG. Para una misma duración del ensayo, el ritmo de germinación media diaria es 1.27 .

La escarificación sulfúrica a concentración de $20 \%$ en otros taxones estudiados pertenecientes a la familia de Leguminosae (como es el caso de Ononis fruticosa y Ononis natrix), resultaba excesivamente agresivo para las semillas; quizá la germinación era un poco más elevada pero daba lugar a mucha contaminación fúngica en placa Petri, y a un desarrollo rápido pero débil de las plántulas, lo cual no nos interesa en absoluto. Sin 


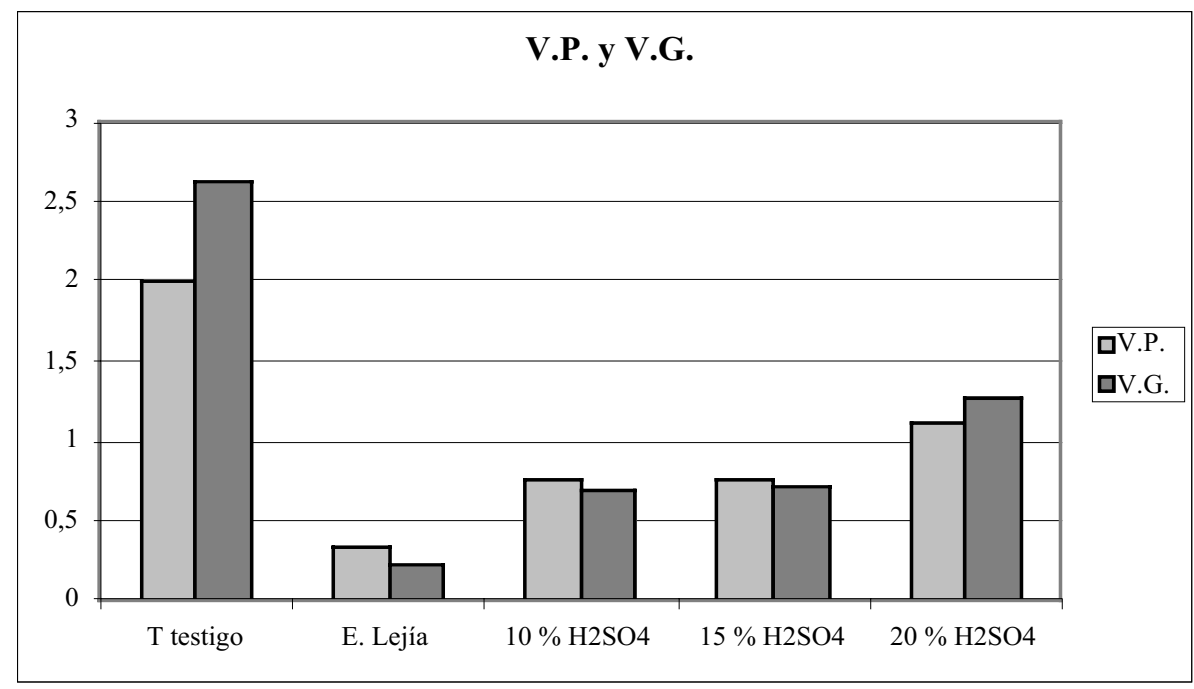

Figura 2. Vigor de Germinación (V.G.) y Valor Pico (V.P.). Germination vigour and high value.

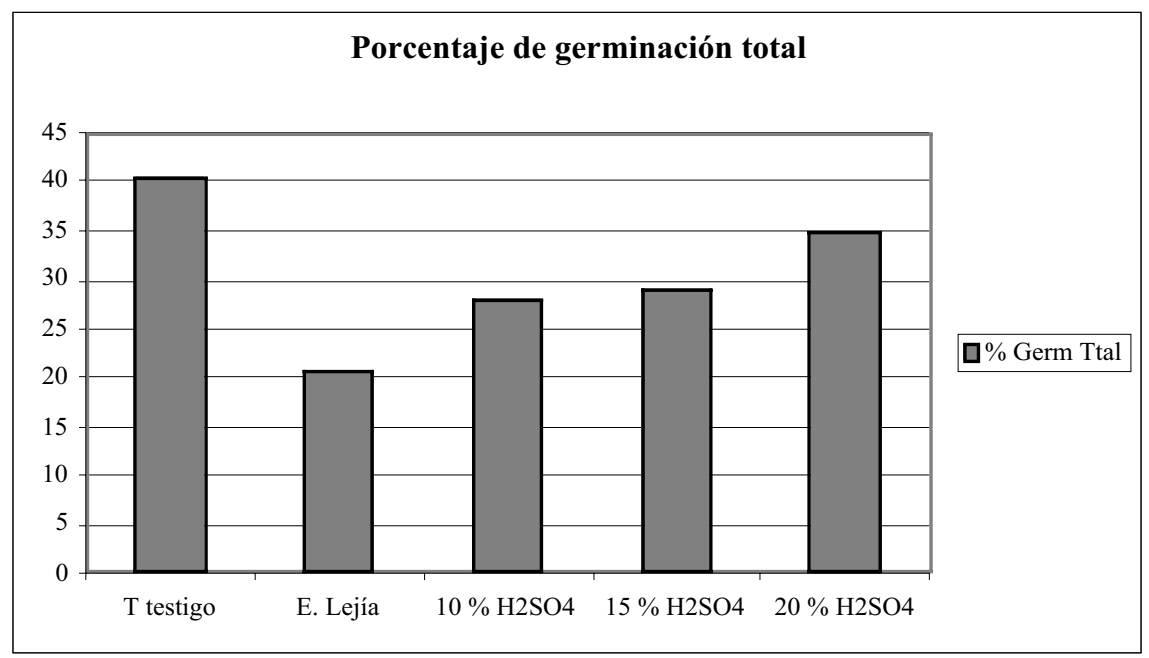

Figura 3. Porcentaje de germinación total. Percentage of total germination. 
embargo en este caso se ha obtendio una respuesta favorable. Es más elevado el porcentaje de germinación total y con un ritmo de germinación media diaria de 1'129, bastante bueno para un mismo periodo de tiempo de ensayo.

Entre los tratamientos de escarificación sulfúrica a concentración de 10 y $15 \%$ no hay grandes diferencias. Respecto a la escarificación con lejía no resulta muy acertado para semillas de este taxón vegetal; siendo los valores obtenidos en la mayoría de parámetros bastante bajos.

Sorprendentemente los tratamientos testigo y de escarificación sulfúrica a la concentración de $20 \%$, han sido los que mejor resultado ofrecen, y de entre ellos el más idóneo es el tratamiento testigo, pues el porcentaje de germinación total es mayor, se inicia antes la germinación del lote, y también son mejores los valores de vigor de germinación y valor pico, así como el ritmo de germinación media diaria.

La agresión que sufren las semillas de este taxon vegetal sometidas a escarificación sulfúrica, da lugar a la rotura de la testa rápidamente; si en dicho momento, cuentan con condiciones de luz, humedad y temperatura óptimas para su desarrollo (condiciones de laboratorio), se desarrollan plántulas fuertes y vigorosas, tanto es así que los hongos no afectan en absoluto las radículas como sucede en otros taxones. Se trata de un momento extremadamente importante, el embrión se desarrolla muy rápidamente.

\section{CONCLUSIONES}

El comportamiento de las semillas de Ononis tridentata, como queda reflejado a lo largo de este estudio es diferente según el ensayo, y el más adecuado de entre ellos es el tratamiento testigo, pues para una misma duración del ensayo se obtiene un porcentaje mayor, mejores valores de VP y VG, y el ritmo de germinación media diaria es bueno. Se ha observado que crece una radícula vigorosa y sin afecciones fúngicas; el porcentaje de emergencia en vivero de este ensayo es bastante bueno y el desarrollo de plántulas y planta adulta adecuado.

No solo nos interesa obtener en germinación porcentajes elevados, sino que la nascencia sea adecuada, planta vigorosa $\mathrm{y}$ fuerte sin afecciones fúngicas y cuyo desarrollo en vivero aunque lento en ocasiones, de lugar a ejemplares sanos. El desarrollo de las plántulas sigue un patrón característico de cada familia, género e incluso especie que es preciso conocer para poder apreciar la forma y maginitud con que las características del terreno pueden influir en la nascencia (Besnier, 1989).

En el medio natural, las semillas de este taxon vegetal se encuentran sometidas a escarificación física en el suelo por fricción, química por la incidencia del sol y por reacciones que pudieran darse en el suelo (ácidos segregados por insectos y otros vegetales). Tales semillas germinan o no sin ningún tipo de tratamiento convencional como los aquí efectuados, se encuentran con unas condiciones de temperatura y humedad fluctuantes y no tan específicas como las de laboratorio. Se ha comprobado en laboratorio que germinan bastante bien sin ningún tratamiento específico (tratamiento testigo en laboratorio).

El ensayo testigo para semillas de Ononis tridentata es el que mejor resultados ha ofrecido.

\section{BIBLIOGRAFÍA}

BESNIER, F. -1989- Semillas. Biología y Tecnología. Ed. Mundi-Prensa.

GÓMEZ-CAMPO, C. et al. -1987-Libro rojo de especies vegetales amenazadas de España 
peninsular e Islas Baleares. Serie Técnica. Ministerio de Agricultura, Pesca y Alimentación. ICONA.

HOPKINGS, E.F. -1923- The behaviour of hard seeds of certains legumes when subjected to conditions favorable to germination. Proceeding Association Official Seeds Analysts of North America 14:46-48.

LAGUNA, E. et al. -1998- Flora vascular rara, endémica y amenazada de la Comunidad Valenciana. Consellería de Medio Ambiente.

MADIA DE CHALUAT, M. -1985- Microorganism present on lucerne (Medicago sativa) seeds. Fitopatologia 20:75-77.

MATEO, G.; CRESPO, M. B. -1998- Manual para la determinación de Flora Valenciana. Monografías de flora Montiberica $n^{\circ} 3$. Alicante-Valencia.

THOMSON, J.R. -1979- Introducción a la tecnología de las semillas. Ed. Acribia.
TRAN, V.N.\& A.K. CAVANAGH -1984Structural aspects of formancy. In: D.R. Murray (Ed.) Seeds physiology 2: 1-44. Academic Press, New York.

Aceptado para su publicación en octubre de 2006

Dirección de los autores. M.C. Escribá Baeza: Vaersa, Ingeniero Técnico Agrícola.. Departamento I.A.S.; E. Laguna Lumbreras: Generalitat Valenciana. Consellería de Territori i Habitatge; Dirección General de Planificación y Gestión del Medio Natural 